RASĀYAN J. Chem.

Vol. 14 | No. 2 |844-854| April - June | 2021 ISSN: 0974-1496 | e-ISSN: 0976-0083 | CODEN: RJCABP http://www.rasayanjournal.com http://www.rasayanjournal.co.in

\title{
INTERACTIONS OF THE CHEMICAL CONSTITUENTS OF Eleutherine americana (AUBL.) MERR. EX K. HEYNE WITH CYCLOOXYGENASE AND H5N1 RNA POLYMERASE: AN In- silico STUDY
}

\author{
S. Damayanti ${ }^{1,2, \bigotimes}$, D. Puspaningrum ${ }^{1}$, H.N. Muhammad ${ }^{3}$, T. Amelia ${ }^{1}$, B. \\ Permana $^{1}$, R. Hartati ${ }^{4}$ and I. Wibowo ${ }^{5}$ \\ ${ }^{1}$ Department of Pharmacochemistry, School of Pharmacy, Institut Teknologi Bandung, J1. \\ Ganesha No.10, Bandung, 40132, Indonesia \\ ${ }^{2}$ University Center of Excellence on Artificial Intelligence for Vision, Natural Language
} Processing, and Big Data Analytics (U-CoE AI-VLB), Institut Teknologi Bandung, Jl. Ganesha No.10, Bandung, 40132, Indonesia

${ }^{3}$ Department of Pharmacology-Clinical Pharmacy, School of Pharmacy, Institut Teknologi Bandung, Jl. Ganesha No.10, Bandung, 40132, Indonesia

${ }^{4}$ Department of Pharmaceutical Biology, School of Pharmacy, Institut Teknologi Bandung, Jl. Ganesha No.10, Bandung, 40132, Indonesia

${ }^{5}$ Department of Physiology Animal Development and Biomedical Science, School of Life Sciences and Technology, Institut Teknologi Bandung, Jl. Ganesha No.10, Bandung, 40132, Indonesia

${ }^{\circledR}$ Corresponding Author: sophi.damayanti@fa.itb.ac.id

ABSTRACT
Eleutherine americana has been shown to have many biological activities such as anti-inflammatory, antipyretic, antiproliferation, antibiotic, and antivirus activities. Many chemical constituents of E. americana have also been identified, of which several may be responsible for these biological activities. In this study, we investigated the potential for these compounds to be developed as anti-inflammatory and antiviral agents through interaction study using molecular docking simulations and in silico toxicity predictions. Four protein target molecules were used in the study; cyclooxygenase-1 (COX-1) and cyclooxygenase-2 (COX-2) were used to screen potential compounds for antiinflammatory activity and polymerase acidic protein $\mathrm{N}$-terminal domain (PAN) and polymerase basic protein 2 (PB2) were used to screen for antiviral activity. One compound, $\beta$-sitosterol, was shown to have a high affinity for COX-1, COX-2, and PB2; $\beta$-sitosterol returned a lower binding free energy score than celecoxib, and a binding free energy score on par with pimodivir. Another compound, compound 9, was also identified as having a higher affinity for PAN than baloxavir. Toxicity predictions of both of these compounds showed none to low toxicity, and thus these compounds have the potential to be developed further as anti-inflammatory and antiviral agents.

Keywords: Eleutherine americana, Anti-inflammation, Antivirus, Molecular Docking, Toxicity Prediction

RASĀYAN J. Chem., Vol. 14, No.2, 2021

\section{INTRODUCTION}

Inflammation is a protective response to tissue injury and infection caused by physical trauma, harmful chemicals, or microbiological substances. ${ }^{1}$ It occurs through several processes: vasodilation of blood vessels increased capillary permeability and swelled (inflammation) tissue cells. This reaction arises due to the release of several biological molecules such as histamine, bradykinin, serotonin, prostaglandins, the complement system reaction products, and various hormonal substances (lymphokines) by sensitized $\mathrm{T}$ cells. ${ }^{2}$ Anti-inflammatory drugs, such as aspirin, celecoxib, and rofecoxib, inhibit the production and release of the inflammatory mediator prostaglandin by inhibiting cyclooxygenase.

Influenza is an infection of the respiratory system caused by viruses, one of which is Influenza A, which causes influenza in humans. According to the FDA, several medications have been developed for the Rasayan J. Chem., 14(2), 844-854(2021)

http://dx.doi.org/10.31788/ RJC.2021.1426117

(c) (i)

This work is licensed under a CC BY 4.0 license. 
RASĀYAN J. Chem.

Vol. 14 | No. 2 |844-854| April - June | 2021

treatment of influenza, namely oseltamivir, zanamivir, and peramivir. However, based on a recent study, several viruses have become resistant to oseltamivir due to mutations in the neuraminidase protein. Therefore, the development of antiviral drugs as inhibitors of Polymerase Basic 2 (PB2) cap-binding, Polymerase Acid (PA) endonuclease and PB1 RNA synthesis have been carried out and are under clinical trials. $^{3}$

Eleutherine americana (EA) has several biological activities such as antibiotic, antivirus, antiinflammation, antipyretic, antiproliferation and cytotoxic effects, and have therefore been used widely by the people of Borneo to deal with heart disease, inflammation, and tumors. ${ }^{4,5}$ To observe the molecular interaction of the major chemical constituents found in EA to enzymes and receptors in the human body, in silico studies can be conducted. Molecular docking studies, a method used in silico studies, attempts to anchor drug candidates into the active site of a known target and predict the affinity of the compounds based on the calculated binding free energy. This type of interaction study is typically employed in the early stage of finding new drugs with the potential to be developed. The degree of toxicity of compounds can also be predicted using an in silico approach. These interaction studies and toxicity predictions provide useful insights in predicting compounds that are effective and relatively non-toxic before the synthesis process so that the research conducted can be done more efficiently in terms of the cost and time spent in the process of new drug discovery.

Therefore, an in silico study, consisting of interaction study and toxicity prediction, was performed to predict the affinity and potential inhibitory activity of the major compounds found in EA on the cyclooxygenase enzymes as one of the targets of commercial anti-inflammatory drugs, and polymerase basic protein 2 (PB2) and polymerase acidic protein N-terminal domain (PAN) of Influenza A virus, which play an important role in the process of transcription and replication of the virus, as a mechanism of influenza antiviral drugs. These compounds exhibited promising results in this study and have the potential to be developed as anti-influenza drug candidates.

\section{Macromolecule Preparation}

\section{EXPERIMENTAL}

Four macromolecules were used as targets for the interaction study (Table-1), namely cyclooxygenase (COX) I, COX II, the polymerase basic protein 2 (PB2) of H5N1, and the polymerase acidic protein Nterminal domain (PAN) of H5N1. Macromolecule structures were obtained from the Protein Data Bank. ${ }^{6}$ The structures were prepared by removing all water and ligand molecules from the protein.

\section{Ligand Preparation}

Thirty-one chemical constituents of Eleutherine americana (EA) were previously identified ${ }^{5,7-10}$ and their structures are drawn (Supplementary Information Table S2) using GaussView 6.0. Quantum mechanical calculations, including geometry optimization and calculations of atomic partial charges, were performed in the Gaussian software package. ${ }^{11}$ The calculations were performed using the density functional theory (DFT) method and the Becke three-parameter Lee-Yang-Parr (B3LYP) hybrid functional at the 6-31G basis set level. Also, four ligands from each of the macromolecule structures (Table-1), used for validation of the docking parameters, as well as four-drug compounds used in anti-inflammation and antiviral therapy, used as reference compounds with which the 31 EA compounds were compared, were prepared in the same way. After geometry optimization, the conformations with the lowest energy were chosen for further interaction study with the macromolecule targets.

\section{Ligand-target Interaction Study through Molecular Docking}

Molecular docking simulations were performed using the AutoDock ${ }^{12}$ software suite to study the interactions between the ligands and the four protein targets. Preparation of the ligands for the docking study was performed using AutoDockTools ${ }^{12}$; partial charges for the protein targets were calculated using the Kollman method ${ }^{13}$, whereas results of the DFT calculations were used to assign partial charges to the atoms of the ligands. Polar hydrogens were also added to the ligand structures. The areas of interest for the docking study were determined by selecting a grid box centered on the protein-bound ligands of each target, and resizing the grid box to fully encompass the binding site, using a grid spacing of $0.375 \AA$. This resulted 
RASĀYAN J. Chem.

Vol. 14 | No. 2 |844-854| April - June | 2021

in grid box sizes of $(40 \times 40 \times 40),(72 \times 40 \times 46),(40 \times 48 \times 58)$, and $(48 \times 48 \times 50)$ for the COX-1, COX-2, PAN, and PB2 targets, respectively. The Lamarckian genetic algorithm was used for the docking simulation, with 100 iterations and 25 million evaluations for each iteration.

Table-1: The Macromolecule Target-bound Ligands used for Validation of the Docking Parameters, and the Drugs used in Anti-inflammation and Antiviral therapy used as Reference. Structures of the Ligands are drawn in Supplementary Information Table S1.

\begin{tabular}{c|c|c|c}
\hline Protein Target & PDB ID & Note & Ligand \\
\hline Cyclooxygenase 1 (COX-1) & 1EQG & $\begin{array}{c}\text { Target-bound crystal } \\
\text { structure \& anti- } \\
\text { inflammatory drug }\end{array}$ & Ibuprofen (IBP) \\
\hline Cyclooxygenase 2 (COX-2) & 1CX2 & $\begin{array}{c}\text { Target-bound crystal } \\
\text { structure }\end{array}$ & $\begin{array}{c}\text { 1-phenylsulfonamide-3- } \\
\text { trifluoromethyl-5- } \\
\text { parabromophenylpyrazole } \\
\text { (S58) }\end{array}$ \\
\hline $\begin{array}{c}\text { H5N1 polymerase basic protein } \\
\text { 2 (PB2) }\end{array}$ & 4CB5 & Target-bound crystal & Celecoxib \\
\hline $\begin{array}{c}\text { H5N1 polymerase acidic protein } \\
\text { N-terminal domain (PAN) }\end{array}$ & 3HW5 & Target-bound crystal \\
structure & $\begin{array}{c}\text { 9-n-(3-carboxy-4- } \\
\text { hydroxyphenyl)-ketomethyl-7- } \\
\text { n-methylguanine (93G) }\end{array}$ \\
\hline & & Antiviral drug & Pimodivir \\
\hline
\end{tabular}

Validation of the docking parameters was performed before docking the test ligands. This was performed by redocking the protein-bound ligands to their respective targets and evaluating the RMSD score between the docking conformation and the crystal structures of the ligands. A high similarity between the two, defined as an RSMD score of less than $2 \AA$, would suggest valid docking parameters. ${ }^{14}$

\section{Toxicity Prediction}

Ligands that showed a higher affinity for the targets compared to the reference ligands, as shown by lower binding free energy calculated from the docking simulations, were assessed for their toxicity using a software-based method. The ADMET Predictor ${ }^{\mathrm{TM}}$ software was used to predict the toxicities of these compounds. Toxicity calculations were performed at physiological $\mathrm{pH}$ for the following parameters: endocrine toxicity, cardiotoxicity, hepatotoxicity, respiratory and skin toxicity, carcinogenicity, and mutagenicity.

\section{Redocking of Reference Compounds to Respective Targets \\ RESULTS AND DISCUSSION \\ The four protein targets used in this study were cyclooxygenase 1 (COX-1), cyclooxygenase 2 (COX-2), the polymerase basic protein 2 (PB2) of $\mathrm{H} 5 \mathrm{~N} 1$, and the polymerase acidic protein $\mathrm{N}$-terminal domain (PAN) of H5N1. The bound ligands in the crystal structure of these proteins were separated and redocked to validate the docking parameters used. The redocking process returned ligand conformations that closely match that of the respective crystal structures, with RMSD values of less than $2 \AA$. Hence, the docking parameters used in the redocking process were deemed valid and were used throughout the study.}

Interaction Study of Eleutherine Americana Compounds with Cyclooxygenase, PB2, and PAN

The interactions of Eleutherine americana (EA) compounds with the four target molecules were studied through molecular docking simulations. The binding free energies calculated from each target-ligand complex were used to rank the compounds and to assess the affinity of the compounds for each target molecule; a low binding free energy corresponds to high affinity and a stable target-ligand interaction. ${ }^{15}$ In this study, COX-1 and COX-2 were selected as targets to assess the potential of EA compounds for preventing inflammation through the inhibition of prostaglandin. COX-2 is involved in the arachidonic acid pathway which results in the synthesis of prostaglandin, an important mediator of inflammation and pain. ${ }^{16}$ Inhibition of COX-2 is the main mechanism of many anti-inflammatory drugs, especially non-steroidal 
RASĀYAN J. Chem.

Vol. 14 | No. 2 |844-854| April - June | 2021

anti-inflammatory drugs (NSAIDs). COX-1, on the other hand, is not directly involved in the inflammatory response, and thus its inhibition typically does not result in anti-inflammatory activity and even causes unwanted side effects. Hence, in this study, EA compounds were screened for high affinity towards COX2, and low affinity towards COX-1, if possible, to discover compounds that selectively target COX-2. To assess the affinity of the EA compounds, ibuprofen and celecoxib were used as reference compounds for binding with COX-1 and COX-2, respectively. These compounds are widely used anti-inflammatory agents that act on the respective target molecules.

In addition to screening for anti-inflammatory potential, we assessed the potential for EA compounds to act as antiviral agents through the inhibition of two protein targets that are essential for viral viability. These proteins are PB2, which is involved in the recognition of the cap of host mRNA, and PAN, which is an endonuclease that cleaves the bonds between the cap and host mRNA. Both of these proteins are involved in the transcription of viral genetic material. Pimodivir and baloxavir ${ }^{17}$, drugs used in antiviral therapy that inhibit PB2 and PAN, respectively, were used as reference compounds.

Table-2: Binding Free Energy of the Four-drug Compounds used as Reference

\begin{tabular}{c|c|c}
\hline Drug Compound & Protein Target & Binding Free Energy $(\mathrm{kcal} / \mathrm{mol})$ \\
\hline Ibuprofen & COX-1 & -7.82 \\
\hline Celecoxib & COX-2 & -10.32 \\
\hline Baloxavir & PAN & -6.23 \\
\hline Pimodivir & PB2 & -8.91 \\
\hline
\end{tabular}

Table-3: Docking Scores, denoted as Binding Free Energy in kcal/mol, of the 31 EA Compounds

\begin{tabular}{|c|c|c|c|c|}
\hline Compound & \multicolumn{4}{|c|}{ Binding Free Energy $(\mathrm{kcal} / \mathrm{mol})$} \\
\hline & $\mathrm{COX}-1$ & $\mathrm{COX}-2$ & PAN & PB2 \\
\hline 1 & -8.12 & -7.56 & -5.36 & -6.50 \\
\hline 2 & -7.66 & -7.47 & -6.12 & -7.64 \\
\hline 3 & -6.77 & -6.65 & -5.05 & -6.56 \\
\hline 4 & -6.83 & -6.77 & -5.09 & -6.61 \\
\hline 5 & -6.90 & -6.78 & -5.02 & -6.74 \\
\hline 6 & -7.56 & -7.38 & -5.62 & -7.43 \\
\hline 7 & -6.50 & -6.38 & -6.34 & -7.12 \\
\hline 8 & -6.69 & -6.59 & -5.02 & -6.40 \\
\hline 9 & -8.14 & -7.59 & -6.71 & -6.49 \\
\hline 10 & -8.46 & -7.22 & -6.50 & -6.73 \\
\hline 11 & -6.82 & -7.07 & -5.85 & -6.85 \\
\hline 12 & -8.07 & -6.94 & -6.57 & -7.25 \\
\hline 13 & -6.96 & -7.43 & -6.08 & -6.94 \\
\hline 14 & -5.99 & -7.85 & -5.12 & -7.24 \\
\hline 15 & -7.70 & -6.97 & -8.08 & -6.87 \\
\hline 16 & -2.33 & -8.93 & -5.01 & -6.37 \\
\hline 17 & -8.34 & -7.85 & -6.48 & -6.75 \\
\hline 18 & -9.11 & -8.51 & -7.08 & -7.76 \\
\hline 19 & -6.71 & -7.01 & -6.55 & -7.37 \\
\hline 20 & -6.09 & -6.87 & -5.55 & -6.11 \\
\hline 21 & -7.69 & -7.39 & -6.96 & -8.02 \\
\hline 22 & -6.41 & -7.75 & -4.57 & -7.63 \\
\hline 23 & -5.83 & -6.57 & -1.42 & -4.35 \\
\hline 24 & -7.11 & -7.10 & -7.02 & -7.36 \\
\hline 25 & -10.66 & -10.66 & -6.53 & -8.19 \\
\hline 26 & -9.45 & -9.58 & -6.21 & -8.59 \\
\hline 27 & -7.43 & -7.21 & -5.22 & -6.91 \\
\hline 28 & -6.95 & -6.56 & -6.31 & -6.98 \\
\hline 29 & 2.12 & -7.98 & -4.57 & -6.03 \\
\hline 30 & -6.90 & -7.42 & -5.10 & -6.81 \\
\hline 31 & -6.36 & -6.60 & -5.89 & -7.30 \\
\hline
\end{tabular}


RASĀYAN J. Chem.

Vol. 14 | No. 2 |844-854| April - June | 2021

Molecular docking simulations of 31 EA compounds to COX-2 resulted in a variety of binding free energies (Table 3). However, only compound $25(-10.66 \mathrm{kcal} / \mathrm{mol})$ was predicted to have a higher affinity towards COX-2 compared to celecoxib $(-10.32 \mathrm{kcal} / \mathrm{mol})$ (Table 2). Other compounds that returned binding free energies close to that of celecoxib were compound $16(-8.93 \mathrm{kcal} / \mathrm{mol}), \mathbf{1 8}(-8.51 \mathrm{kcal} / \mathrm{mol})$, and $\mathbf{2 6}(-9.58$ $\mathrm{kcal} / \mathrm{mol})$. Meanwhile, compounds that are unique to EA, namely compounds $\mathbf{1 - 5}$ and $\mathbf{1 1}$, did not result in higher affinity for COX-2, but also returned low binding free energies. To confirm whether these compounds did indeed have a lower affinity for COX-2 compared to celecoxib, an independent t-test was performed. The results show statistically significant differences $(\mathrm{p}<0.05)$ between the binding free energies of these compounds and that of celecoxib, except for compound 25.

According to D'Mello et al. ${ }^{18}$, COX-2 inhibitors interact with their target at three main binding sites: (1) a hydrophobic pocket with amino acid residues Tyr385, Trp387, Phe518, Tyr248, and Leu 352; (2) hydrophilic amino acid residues Arg120, Glu524, and Tyr355, which are located at the entrance of the binding site; and (3) a side pocket with His90, Arg513, and Val523. Celecoxib interacts with COX-2 by forming hydrogen bonds with four amino acid residues, three of which are residues identified as crucial to COX-2 inhibitory activity (Fig.-1a). In addition to hydrogen bonds, other important interactions that formed were $\pi$-sulfur and $\pi$-cation bonds, which further stabilized the celecoxib-COX-2 complex. Interestingly, compound $\mathbf{2 5}$ did not form any strong hydrogen bonds with COX-2, but the strong interactions between it and the binding site were mostly contributed to by hydrophobic interactions, especially $\pi$-alkyl bonds (Fig.1b). It formed non-bonded interactions with Trp387, Tyr385, Leu352, Phe518, Tyr355, and Val523, which are all amino acid residues that are part of the three main binding sites for COX-2 inhibition. This is more than that of celecoxib-COX-2 interactions, which may explain why compound 25's affinity is on par with celecoxib.

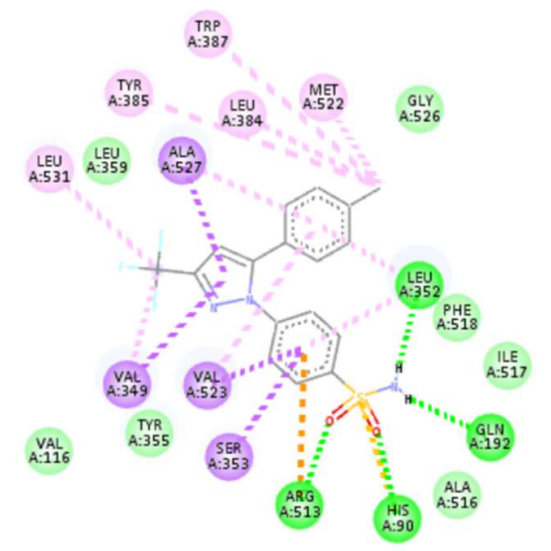

a

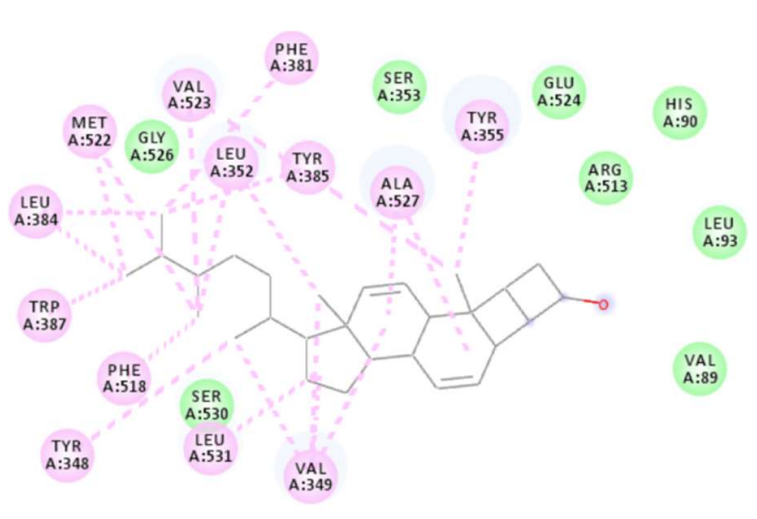

b

Fig.-1: (a) Binding of Celecoxib at the COX-2 binding site results in the formation of several important interactions: four hydrogen bonds (dotted green lines) with the Arg513, His90, Gln192, and Leu352 amino acid residues; a $\pi$ sulfur bond (dotted yellow line) with His90; and four $\pi$-cation bonds (dotted purple lines) with Ser353, Val523, Val349, and Ala527. (b) Compound 25 bound strongly with COX-2 at the binding site by forming numerous, strong hydrophobic interactions (dotted pink lines).

The six compounds unique to EA formed several hydrogen bonds and hydrophobic interactions with amino acid residues of the COX-2 binding site (Fig.-S1 in the Supplementary Information). However, the binding free energy of the interaction between these compounds and COX-2 was not as low as that of celecoxib; these EA compounds resulted in free energy values in the range of -6.65 to $-7.56 \mathrm{kcal} / \mathrm{mol}$, compared to celecoxib's $-10.57 \mathrm{kcal} / \mathrm{mol}$. This may be explained by the fact that celecoxib cannot only form more hydrogen bonds with the COX-2 binding site than these EA compounds but also form more interactions in general.

Interactions between the $31 \mathrm{EA}$ compounds with COX-1 show that only eight compounds have a higher affinity for COX-1 compared to the reference compound, ibuprofen. Similar to its affinity for COX-2, 
RASĀYAN J. Chem.

Vol. 14 | No. 2 |844-854| April - June | 2021

compound 25 showed a higher affinity for COX-1 compared to the reference, although here it is much higher $(-10.32 \mathrm{vs}-7.82 \mathrm{kcal} / \mathrm{mol})$, which suggests non-selective binding to cyclooxygenase. Thus, compound $\mathbf{2 5}$ has the potential to be explored further as an anti-inflammatory drug candidate.

Ibuprofen forms two strong hydrogen bonds with COX-1, both with the Arg120 residue, at 1.674 and 1.750 $\AA$ (Figure 2a). Also, it forms a $\pi$-sigma bond with Ile523. As for the interaction between compound $\mathbf{2 5}$ and COX-1, similar to its interaction with COX-2, numerous hydrophobic interactions are the primary reason for the high affinity exhibited (Figure $2 b$ ). Of the six compounds unique to EA, with the sole exception of compound $\mathbf{1}$, all resulted in lower affinities towards COX-1 compared to ibuprofen; compound $\mathbf{1}$ resulted in binding free energy of $-8.12 \mathrm{kcal} / \mathrm{mol}$ while ibuprofen returned $-7.82 \mathrm{kcal} / \mathrm{mol}$. However, this does not necessarily mean that compound $\mathbf{1}$ shows the potential for anti-inflammatory activity since COX-1 is not involved directly in the inflammation process.

In addition to the high predicted affinity of compound 25 to COX-1 and COX-2, as well as the intermolecular interactions formed, it is interesting to note this may be because compound $\mathbf{2 5}$ ( $\beta$-sitosterol) is a steroid compound with high similarity to corticosteroids, which are known, anti-inflammatory agents. Corticosteroids exhibit anti-inflammatory activity through the inhibition of phospholipase A2 and preventing the production of arachidonic acid ${ }^{19}$.

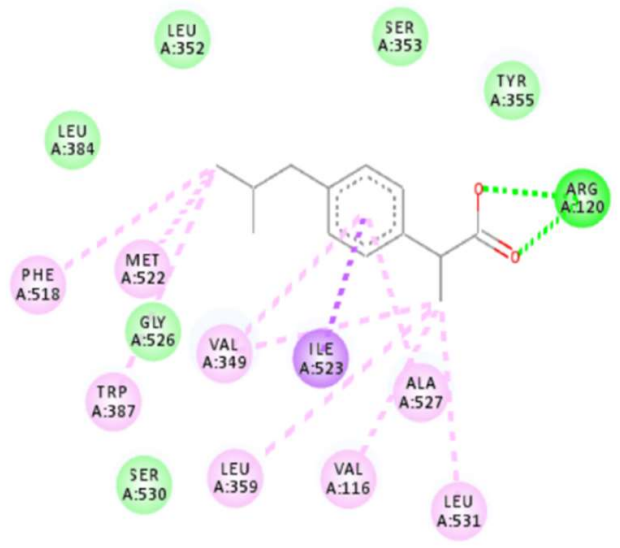

a

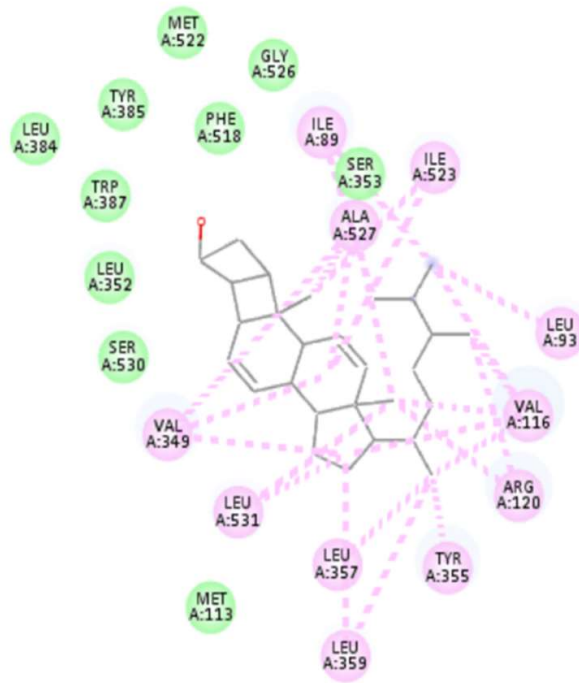

b

Fig.-2: (a) Ibuprofen Forms two hydrogen bonds (dotted green lines) with COX-1 and many hydrophobic interactions (dotted pink lines). (b) Interaction of compound 25 with COX-1. Although no hydrogen bonds were observed, many hydrophobic interactions between compound 25 and COX-1 amino acid residues were formed.

The docking results of compounds unique to EA (compounds 1-5 and 11) suggest that they do not have a higher affinity for COX-2 compared to the reference compound used, celecoxib. However, these compounds were shown to have anti-inflammatory properties, albeit through a different mechanism of action, namely the inhibition of inducible nitrous oxide synthase (iNOS) ${ }^{20}$.

Out of the 31 compounds used in the docking study, 12 returned predicted affinities towards PAN higher than that of the reference compound, baloxavir (Fig.-3). Toxicity predictions were performed for these 12 compounds, and the least toxic compounds were screened and used for further analysis (Table-4). Four compounds with a high affinity for PAN were determined to be the least toxic, namely compounds $\mathbf{9 , 1 0}$, 17, and 25. However, compound 9 is predicted to have the highest affinity for PAN, as shown by the docking score, and as such was chosen as the potential candidate for further development. Compound 9 exhibited a lower binding free energy $(-6.71 \mathrm{kcal} / \mathrm{mol})$ when docked to the binding site of PAN compared to that of baloxavir $(-6.23 \mathrm{kcal} / \mathrm{mol}$ ) (Fig.-3). It formed five hydrogen bonds with amino acid residues of the PAN binding site, namely two bonds with Lys234 and one bond each with Tyr130, Arg124, His41 (Figure 3b). Also, it formed two $\pi$-cation bonds with His41, strengthening their interaction. Similarly, 
RASĀYAN J. Chem.

Vol. 14 | No. 2 |844-854| April - June | 2021

baloxavir interacts with the binding site of PAN by forming three hydrogen bonds and two $\pi$-cation bonds. Interestingly, two of these hydrogen bonds are formed with the same amino acid residues as those compound 9 formed bonds with, namely Tyr 130 and Lys 134 (Fig.-3a), and the two $\pi$-cation bonds formed are also with His41. However, the fewer number of hydrogen bonds formed by baloxavir (three) compared to that formed by compound 9 (five) with PAN may explain the slightly higher affinity for PAN of compound 9. None of the six compounds unique to EA (compounds 1-5 and 11) showed a higher affinity for PAN compared to baloxavir (Table-3). Although these compounds interacted with the PAN binding site by forming hydrogen bonds and $\pi$-cation bonds (Figure S2, Supplementary Information), similar to baloxavir and compound $\mathbf{9}$, the number of bonds formed were fewer and this may be a contributing factor to the lower affinity.

Docking simulations of the 31 test compounds on PB2 did not result in any compound with a higher affinity for PB2 compared to the reference compound, pimodivir (Fig.-3). However, three compounds resulted in binding free energies that were similar to that of pimodivir $(-8.91 \mathrm{kcal} / \mathrm{mol})$, namely compound $21(-8.02$ $\mathrm{kcal} / \mathrm{mol}), 25(-8.19 \mathrm{kcal} / \mathrm{mol})$, and $26(-8.59 \mathrm{kcal} / \mathrm{mol})$. An independent t-test statistical test was performed to determine whether these compounds exhibited a different affinity for PB2 compared to pimodivir, and the results show that the binding free energies of these three compounds were not significantly different from that of pimodivir, with a confidence interval of $95 \%$.

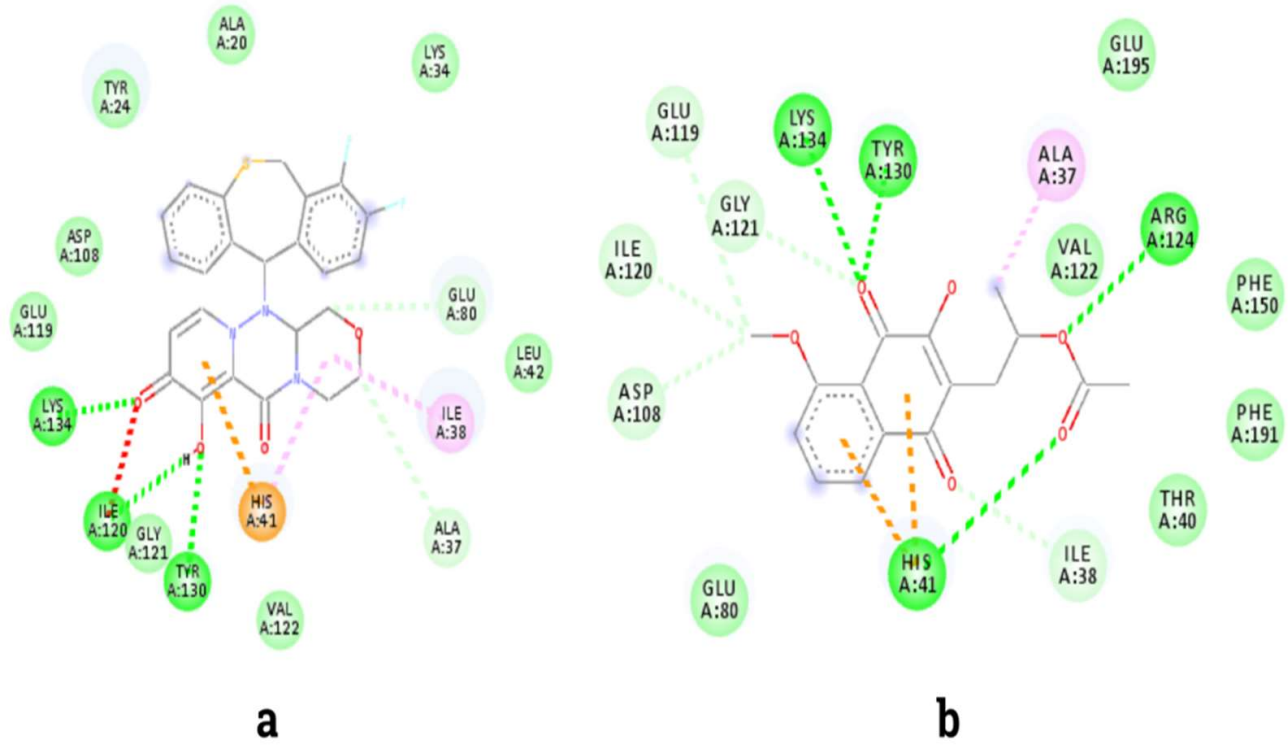

Fig.-3:(a) Baloxavir Forms three hydrogen bonds (dotted green lines) with amino acid residues of the PAN binding site: Ile120, Tyr130, and Lys134; and two $\pi$-cation bonds with His41. (b) Compound 9 is predicted to have a higher affinity for PAN compared to baloxavir. It forms four hydrogen bonds (dotted green lines) with His41, Arg124, Tyr130, and Lys 134; and two $\pi$-cation bonds with His41.

Toxicity predictions were performed on compounds $\mathbf{2 1}, \mathbf{2 5}$, and $\mathbf{2 6}$ to select the least toxic compound for further analysis (Table-4), and the results show that compound $\mathbf{2 5}$ is predicted to be the least toxic, and thus was selected for further analysis.

Pimodivir interacts strongly with PB2 due to the formation of three hydrogen bonds with the amino acid residues Arg355, His357, and Glu361 (Fig.-4a). It also forms six $\pi$ - $\pi$ stacked interactions with Phe323, His357, and Phe404, which contribute significantly to the overall strength of the interaction. In addition to the strong interactions formed between pimodivir and the PB2 binding site, pimodivir's chemical structure greatly determines its strong inhibitory activity towards PB2. Pimodivir contains a 7-azaindole structure, which has been shown in vivo experiments to be the main factor for influenza A antiviral activity ${ }^{21}$. Unlike pimodivir, compound $\mathbf{2 5}$ forms a single hydrogen bond with the PB2 binding site, and no $\pi$ - $\pi$ interactions (Fig.-4b). However, it interacts strongly with PB2, having a similar affinity with pimodivir. This may be 
RASĀYAN J. Chem.

Vol. 14 | No. 2 |844-854| April - June | 2021

explained by the fact that compound $\mathbf{2 5}$ forms many hydrophobic interactions with many amino acid residues of the PB2 binding site.

\section{In-silico Toxicity Predictions}

Molecular docking simulations of the EA compounds to COX-2 showed that compound $\mathbf{2 5}$ was the only compound predicted to have the same affinity for COX-2 as celecoxib. Thus, in addition to the six compounds unique to EA, compound $\mathbf{2 5}$ was used in the in silico toxicity prediction.

Although the results of the toxicity prediction show that compound $\mathbf{2 5}$ may increase gamma glutamine transferase (GGT) and lactate dehydrogenase (LDH) concentrations, predictions of the TOX_AlkPhos, TOX_SGOT, and TOX_SGPT values were negative (non-toxic) (Table-4). Meanwhile, all of the compounds unique to EA except for compound $\mathbf{1 1}$ returned negative results for prediction of hepatotoxicity; compound 11 is predicted to be able to increase LDH and SGOT concentrations, resulting in potential hepatotoxicity. Furthermore, these compounds were predicted to be non-toxic for endocrine toxicity, although compounds $\mathbf{5}$ and $\mathbf{1 1}$ may be able to slightly increase androgen concentrations. Likewise, predictions of cardiotoxicity, respiratory toxicity, and skin irritation activity returned negative results for these compounds.

Prediction of the carcinogenicity of the six compounds unique to EA and compound $\mathbf{2 5}$ was also performed; the prediction parameter used was the tumor dose $50\left(\mathrm{TD}_{50}\right)$ in rats and mice, which shows the dose required for inducing tumors in $50 \%$ of the sample tested. The results returned high $\mathrm{TD}_{50}$ values, suggesting that these compounds are not carcinogenic ${ }^{22}$. Typically, carcinogenic compounds have a $\mathrm{TD}_{50}$ value of less than $25 \mathrm{mg} / \mathrm{kg} /$ day and $4 \mathrm{mg} / \mathrm{kg} /$ day for mice and rats, respectively (Table-4). The mutagenic potential of the compounds was assessed by predicting the results of the Ames Test using different strains of Salmonella typhimurium (Table-4). A compound is predicted to be mutagenic if it returns a positive Ames Test result with at least one strain in each of the whole compound and metabolite tests. The results show that only compound 25 is predicted to be non-mutagenic. Considering the overall results of all toxicity predictions performed, compound $\mathbf{2 5}$ is forecast to be less toxic compared to the six compounds unique to EA.

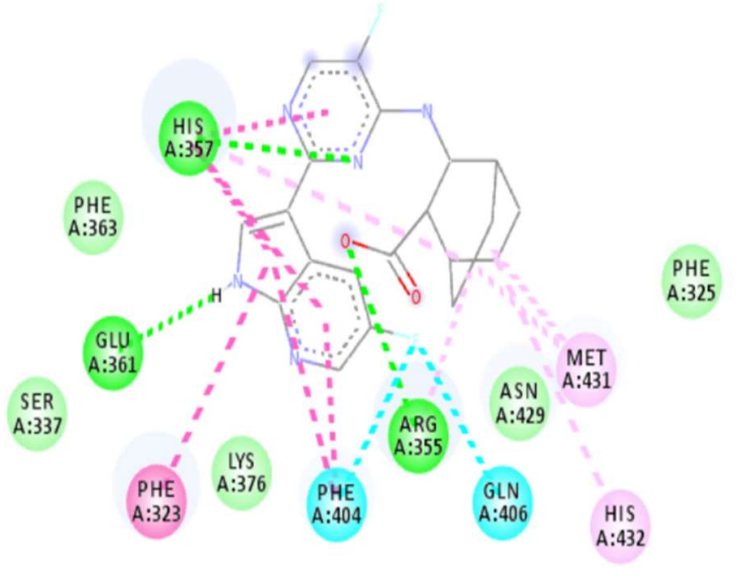

a

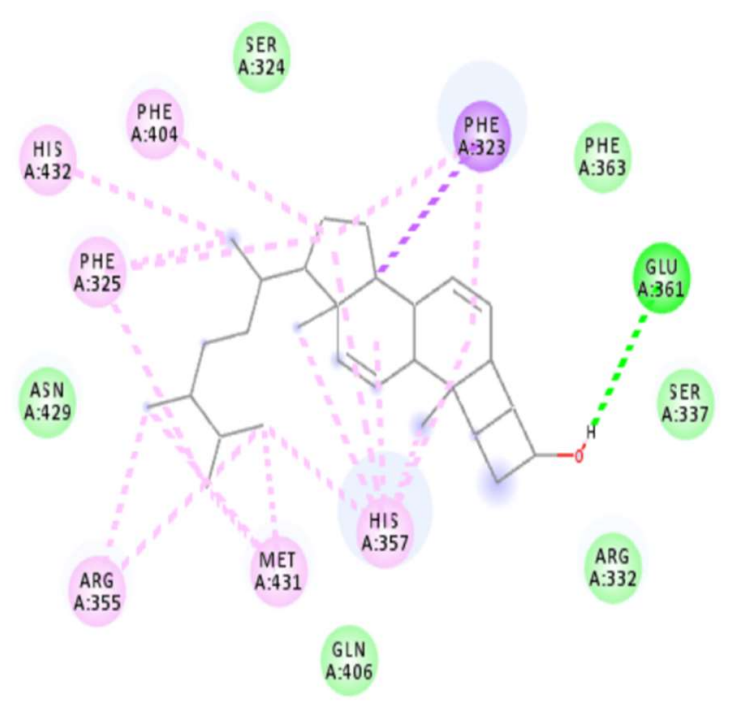

b

Fig.-4: (a) Pimodivir Forms three hydrogen bonds (dotted green lines) with Arg355, His357, and Glu361 at the PB2 binding site, and six $\pi$ - $\pi$ stacked interactions (dotted purple lines) with Phe323, His357, and Phe404. (b) Compound 25 forms a single hydrogen bond (dotted green line) with Glu361 at the PB2 binding site but interacts strongly through many hydrophobic interactions (dotted pink lines).

The results of the docking study showed that 12 tested compounds were predicted to have a higher affinity for PAN compared to baloxavir. Toxicity predictions were performed for these compounds to assess their 
RASĀYAN J. Chem.

Vol. 14 | No. 2 |844-854| April - June | 2021

endocrine toxicity, cardiotoxicity, hepatotoxicity, skin irritation potential, respiratory toxicity, carcinogenicity, and mutagenicity (Table-4). The endocrine toxicity and cardiotoxicity predictions returned negative for all 12 compounds. Meanwhile, compound 12 returned positive for skin irritation and respiratory toxicity, and compounds 24 and 28 returned positive for respiratory toxicity and skin irritation predictions, respectively. Therefore, we excluded these compounds from further study. From the remaining compounds, four of them namely compounds $9, \mathbf{1 0}, \mathbf{1 7}$, and 25 , were predicted to be the least toxic compounds.

The molecular docking results show that compounds $\mathbf{2 1 , 2 5}$, and $\mathbf{2 6}$ were found to have affinities for PB2 on par with that of pimodivir. Toxicity predictions were performed on these compounds to assess their endocrine toxicity, cardiotoxicity, hepatotoxicity, skin irritation potential, respiratory toxicity, carcinogenicity, and mutagenicity (Table-4). The results show that all three compounds were predicted to be non-toxic for respiratory, skin irritation, and cardiotoxicity. As for hepatotoxicity predictions, compound 21 returned positive for three of the five parameters, while compounds $\mathbf{2 5}$ and $\mathbf{2 6}$ returned positive for two parameters. Also, compound $\mathbf{2 6}$ returned positive for endocrine toxicity, and none of the compounds are predicted to be carcinogenic or mutagenic, except for compound 21, which is predicted to be mutagenic towards the 102+wp2 and 98 strains of $S$. typhimurium. Overall, of the three compounds tested, compound 25 is predicted to be the least toxic.

Table-4: In silico Toxicity Prediction for Hepatotoxicity, Carcinogenicity, Mutagenicity, and Endocrine Toxicity of Compounds of Interest.

\begin{tabular}{|c|c|c|c|c|c|c|c|c|c|c|c|c|c|c|c|c|c|}
\hline \multirow[t]{2}{*}{ Compound } & \multicolumn{5}{|c|}{ Hepatotoxicity Parameters } & \multicolumn{2}{|c|}{ Carcinogenicity } & \multicolumn{3}{|c|}{$\begin{array}{c}\text { Mutagenicity } \\
\text { (Whole } \\
\text { Compound) }\end{array}$} & \multicolumn{4}{|c|}{$\begin{array}{l}\text { Mutagenicity } \\
\text { (Metabolite) }\end{array}$} & \multicolumn{3}{|c|}{$\begin{array}{l}\text { Endocrine } \\
\text { Toxicity }\end{array}$} \\
\hline & 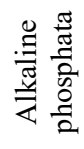 & 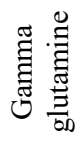 & 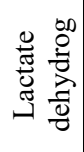 & $\begin{array}{l}5 \\
0 \\
\text { ¿ }\end{array}$ & $\stackrel{5}{0}$ & $\stackrel{\tilde{E}}{\approx}$ & $\begin{array}{l}0 \\
\mathscr{0} \\
\sum_{\Sigma}^{0}\end{array}$ & $\begin{array}{c}\hat{n} \\
\frac{n}{7} \\
\frac{1}{a}\end{array}$ & $\stackrel{\infty}{\sigma}$ & 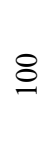 & 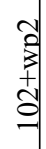 & 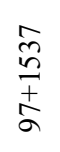 & $\infty$ & 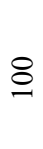 & 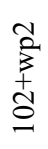 & 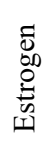 & 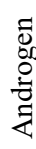 \\
\hline 1 & - & - & - & - & - & 117.41 & 1625.43 & - & + & - & + & - & - & - & - & - & - \\
\hline 2 & - & - & - & - & + & 192.47 & 1476.02 & - & + & + & - & - & - & - & - & - & - \\
\hline 3 & - & - & - & + & + & 198.84 & 1245.97 & - & + & + & - & - & - & + & - & - & - \\
\hline 4 & - & - & - & + & + & 205.90 & 1365.34 & - & + & + & - & - & - & + & - & - & - \\
\hline 5 & - & - & + & - & - & 198.67 & 1028.56 & + & + & - & - & + & - & - & - & - & - \\
\hline 7 & - & - & + & - & - & 400.65 & 2104.57 & + & + & - & - & + & - & - & - & - & - \\
\hline 9 & - & - & - & - & - & 29.35 & 5559.08 & - & - & - & - & - & - & - & - & - & - \\
\hline 10 & - & - & - & - & - & 38.47 & 3574.22 & - & - & - & - & - & - & - & - & - & - \\
\hline 11 & - & - & + & + & - & 412.12 & 518.76 & + & + & - & - & + & - & + & - & - & - \\
\hline 12 & + & + & - & + & + & 448.36 & 29.78 & - & - & - & - & - & - & + & - & - & - \\
\hline 15 & - & - & + & + & + & 7.18 & 22761.54 & - & - & - & - & - & - & - & - & - & - \\
\hline 17 & - & + & + & - & - & 424.95 & 736.44 & - & - & - & - & - & - & - & - & - & - \\
\hline 18 & - & + & - & + & + & 117.41 & 1211.03 & - & - & - & - & - & - & - & - & - & - \\
\hline 19 & - & - & - & - & - & 312.13 & 702.81 & - & - & - & + & - & + & - & - & - & - \\
\hline
\end{tabular}


RASĀYAN J. Chem.

Vol. 14 | No. 2 |844-854| April - June | 2021

\begin{tabular}{c|c|c|c|c|c|c|c|c|c|c|c|c|c|c|c|c|c|c}
\hline 21 & - & + & - & + & + & 143.77 & 1015.59 & - & - & - & + & - & + & - & - & - & - \\
\hline 24 & - & - & - & + & - & 686.03 & 2536.87 & - & - & - & - & + & - & + & + & - & - \\
\hline 25 & - & + & + & - & - & 424.92 & 736.68 & - & - & - & - & - & - & - & - & - & - \\
\hline 26 & + & + & - & - & - & 401.65 & 281.88 & - & - & - & - & - & - & - & - & - & 0.1 \\
\hline 28 & - & - & - & - & - & 678.49 & 1864.96 & - & - & - & - & - & - & - & + & - & - \\
\hline
\end{tabular}

Note:

$(+)$ predicted to have a toxic effect, $(-)$ predicted to not have any effect.

Carcinogenicity stated in tumor dose 50 (TD50) ( $\mathrm{mg} / \mathrm{kg}$ body weight/day orally).

\section{CONCLUSION}

We have performed an in silico study investigating the potential for 31 compounds found in Eleutherine americana to be developed as anti-inflammatory and antiviral agents. Our results show that $\beta$-sitosterol (compound 25) has a high affinity for cyclooxygenase-1 and cyclooxygenase-2, as calculated through molecular docking simulations, higher than that of celecoxib. Furthermore, we found that (2S)-1-(3hydroxy-5-methoxy-1,4-dioxo-1,2,3,4-tetrahydronaphthalen-2-yl) propan-2-yl acetate (compound 9) and $\beta$-sitosterol also have a high affinity for the polymerase acidic protein N-terminal domain (PAN) and polymerase basic protein 2 (PB2) of H5N1 influenza, respectively. Both compounds were predicted to be non-toxic through in silico toxicity predictions.

\section{ACKNOWLEDGEMENT}

This research was supported by the Research, Innovation, and Community Service Program (P3MI) of Institut Teknologi Bandung 2020.

\section{REFERENCES}

1. M. J. Mycek, R. A. Harvey, and P. A. Champe, Pharmacology $2^{\text {nd }}$ edition, Lippincott Williams \& Wilkins, Philadelphia (2001).

2. A. C. Guyton and J. Hall, Guyton and Hall Textbook of Medical Physiology, Elsevier, Philadelphia (2006).

3. A. Stevaert and L. Naesens, Medicinal Research Reviews, 36(6), 1127(2016), DOI: $10.1002 /$ med.21401

4. P. Babula, V. Adam, L. Havel, and R. Kizek, Current Pharmaceutical Analysis, 5(1), 47(2009), DOI: $10.2174 / 157341209787314936$

5. E. M. Kuntorini and L. H. Nugroho, Biodiversitas Journal of Biological Diversity, 11(2) (2010), DOI:10.13057/biodiv/d110210

6. H. M. Berman, J. Westbrook, Z. Feng Z, G. Gilliland, T. N. Bhat, H. Weissig, I. N. Shindyalov, and P. E. Bourne, Nucleic Acids Research, 28(1), 235(2000), DOI:10.1093/nar/28.1.235

7. E. M. Kuntorini, Biodiversitas Journal of Biological Diversity, 17(1), 229(2016), DOI:10.13057/biodiv/d170133

8. P. Babula, R. Mikelová, D. Potěšil, V. Adam, R. Kizek, L. Havel, and Z. Sladký, Biomedical Paper, (149), 25(2005).

9. T. Robinson T, Kandungan Organik Tumbuhan Tinggi, translated by Kosasih Padmawinata, Penerbit ITB, Bandung (1995).

10. R. B. Herbert, Biosintesis Metabolit Sekunder, translated by Bambang Srigandono, IKIP Semarang Press, Semarang (1995).

11. M. J. Frisch, G. W. Trucks, H. B. Schlegel, G. E. Scuseria, M. A. Robb, J. R. Cheeseman, G. Scalmani, et al. Gaussian 09, Revision A.02, Gaussian, Inc., Wallingford CT (2016).

12. G. M. Morris, R. Huey, W. Lindstrom, M. F. Sanner, R. K. Belew, D. S. Goodsell, and A.J. Olson, Journal of Computation Chemistry, 30(16), 2785(2009), DOI:10.1002/jcc.21256

13. B. H. Besler, K. M. Merz, and P. A. Kollman, Journal of Computional Chemistry, 11(4), 431(1990), DOI: $10.1002 /$ jcc.540110404 
RASĀYAN J. Chem.

Vol. 14 | No. 2 |844-854| April - June | 2021

14. G. M. Morris, D. S. Goodsell, R. S. Halliday, R. Huey, W. E. Hart, R. K. Belew, and A. J. Olson, Journal of Computation Chemistry, 19(14), 1639(1998), DOI:10.1002/(SICI)1096987X(19981115)19:14<1639::AID-JCC10>3.0.CO;2-B

15. T. M. Austin, D. P. Nannemann, S. L. Deluca, J. Meiler, and E. Delpire, Journal of Structural Biology, 187(1), 58(2014), DOI:10.1016/j.jsb.2014.05.001

16. A. Kawabata, Biological \& Pharmaceutical Bulletin, 34(8), 1170(2011), DOI:10.1248/bpb.34.1170

17. N. Kawaguchi, H. Koshimichi, T. Ishibashi, and T. Wajima, Clinical Drug Investigation, 38(11), 1053(2018), DOI: 10.1007/s40261-018-0697-2

18. P. D'Mello, M. K. Gadhwal, U. Joshi, and P.Shetgiri, International Journal of Pharmacy and Pharmaceutical Science, 3(4), 33(2011).

19. S. T. V. Raghavamma, N. Rama Rao, and G. Devala Rao, Journal of Genetic Engineering and Biotechnology, 14(1), 211(2016), DOI:10.1016/j.jgeb.2015.11.002

20. M. Insanu, S. Kusmardiyani, and R. Hartati, Procedia Chemistry, 13, 221(2014), DOI: $10.1016 /$ j.proche. 2014.12 .032

21. A. Pflug, S. Gaudon, P. Resa-Infante, M. Lethier, S. Reich, W. M. Schulze, and S. Cusack, Nucleic Acids Research, 46(2), 956(2018), DOI:10.1093/nar/gkx1210

22. L. S. Gold, C. B. Sawyer, R. Magaw, G. M. Backman, M. de Veciana, R. Levinson, N. K. Hooper, W. R. Havender, L. Bernstein, R. Peto, M. C. Pike, and B. N. Amestt, Environmental Health Perspectives, 58, 9-319 (1984), DOI:10.1289/ehp.84589

[RJC-6117/2020] 